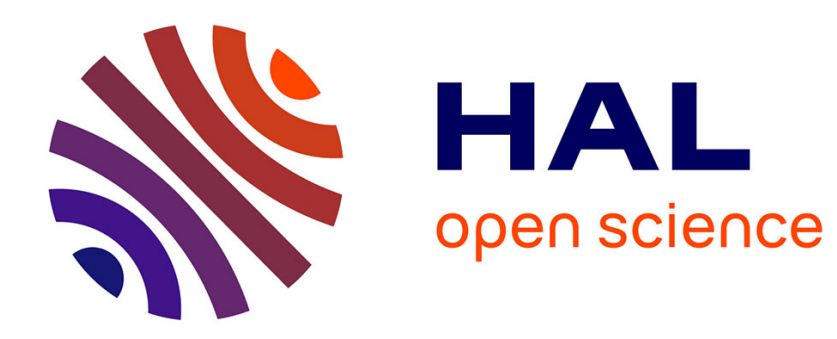

\title{
Tracking Meteorological Structures through Curves Matching Using Geodesic Paths
}

\author{
Isaac Cohen, Isabelle Herlin
}

\section{To cite this version:}

Isaac Cohen, Isabelle Herlin. Tracking Meteorological Structures through Curves Matching Using Geodesic Paths. [Research Report] RR-3191, INRIA. 1997. inria-00073498

\section{HAL Id: inria-00073498 \\ https://hal.inria.fr/inria-00073498}

Submitted on 24 May 2006

HAL is a multi-disciplinary open access archive for the deposit and dissemination of scientific research documents, whether they are published or not. The documents may come from teaching and research institutions in France or abroad, or from public or private research centers.
L'archive ouverte pluridisciplinaire HAL, est destinée au dépôt et à la diffusion de documents scientifiques de niveau recherche, publiés ou non, émanant des établissements d'enseignement et de recherche français ou étrangers, des laboratoires publics ou privés. 


\section{Tracking Meteorological Structures through Curves Matching Using Geodesic Paths}

Isaac COHEN, Isabelle HERLIN

$\mathbf{N}^{\circ} 3191$

June 1997

THËME 3

apport

derecherche 



\title{
Tracking Meteorological Structures through Curves Matching Using Geodesic Paths
}

\author{
Isaac COHEN, Isabelle HERLIN \\ Thème 3 - Interaction homme-machine, \\ images, données, connaissances \\ Projet AIR
}

Rapport de recherche $\mathrm{n}^{\circ} 3191$ - June 1997 - 999999 pages

\begin{abstract}
This paper is concerned with the problem of tracking clouds structures like vortices in meteorological images. For this purpose we characterize the deformation between two successive occurrences, by matching their two boundary curves. Our approach is based on the computation of the set of paths connecting the two curves to be matched. It minimizes a cost function which measures the local similarity of the two curves. These matching paths are obtained as geodesic curves on this cost surface. Moreover our method allows to consider complex curves of arbitrary topology since these curves are represented through an implicit function rather than through a parameterization. Experimental results are given to illustrate the properties of the method in processing synthetic and then meteorologic remotely-sensed data.
\end{abstract}

Key-words: Curves matching, Image sequence analysis, Geodesic distance computation, Eulerian formulation.

(Résumé : tsvp) 


\section{Suivi de structures météorologiques à l'aide de chemins géodésique}

Résumé : Dans cet article nous nous intéressons au suivi de structures nuageuses telles que les vortex dans les images météorologiques satellitaires. Pour cela, nous caractérisons la déformation entre les deux occurrences en appariant les contours des structures.

Notre approche est basée sur le calcul d'un ensemble de chemins reliant les courbes à mettre en correspondance. Ces chemins ou trajectoires minimisent une fonction de coût mesurant la similarité locale des deux courbes. Ils représentent des géodésiques de la surface mesurant la fonction de coût.

De plus, l'approche proposée permet d'apparier des courbes de topologie complexe et variable, car les courbes sont représentées à l'aide d'une fonction implicite.

Des résultats expérimentaux sont donnés en fin d'article pour illustrer l'appariement de structures synthétiques et de structures issues d'imagerie météorologique satellitaire. 


\section{Introduction}

Images sequences obtained from environmental satellites platforms present a new challenge for geosciences and computer vision. The wide range of remote sensors allow to characterize natural phenomena and infer some physical measurements used in atmospheric models. For example, meteorologist use clouds in meteosat images as landmarks for estimating their motion and characterize some subtropical phenomena. Several approaches can be used to track these phenomena: optical flow methods [4] or a method based on pointwise tracking of moving structures like vortices and fronts [1]. In this paper, we develop a new method for pointwise tracking of structures by matching their contours. Hence, the deformation between two temporal occurrences will be obtained through a set of trajectories provided by the matching process. Our method is based on the computation of a set of paths connecting the two curves to be matched. Each path minimize a cost function which measures the local similarity between the starting and ending points of the path. In the next subsection we explain how our method differs from classical ones and define its properties.

\subsection{Related Works and Definition of our method}

Several authors proposed methods based on invariant geometrical properties in order to measure the similarity between the curves. Often these models rely on curvature information $[3,13]$ and are applied in case of rigid motion or when the small deformation hypothesis is valid.

When this last assumption is no more satisfied, curvature measure is not reliable. Some other approaches are based on a parameterization of the deformation in order to derive a similarity measure: Berroir et al [1] proposed a method based on the geometry of the surface generated by the two curves to be matched. This method performs well as long as the surface remains smooth and differentiable. Unfortunately this approach can not handle changes in topology nor large deformations since it uses a uniform parameterization.

In this paper we present a new method, which computes the set of paths joining the curves to be matched, within the applicative framework of atmospheric structure matching. This applicative framework will mainly be used to justify our different hypothesis and to present experimental re-

$\operatorname{RR} n^{\circ} 3191$ 
sults at the end of the paper.

Our approach defines a set of paths starting from the first curve (the source $S$ ) and ending at the second curve (the destination $D$ ). These paths are computed by minimizing a cost function which measures the local similarity of the curves $S$ and $D$, and they are defined as geodesics of this cost function surface.

In order to satisfy the requirements of our application, we consider the following approach:

- The cost function is defined through a graph surface and measures, as we previously explained, the similarity between the source and destination areas. As this function will be defined over the whole 2D plane, computation may be achieved independently of the topology of the curves. This surface is the graph surface on which the connecting paths are computed.

- We choose to define the source and destination curves through two level set functions. This allows to consider a large family of curves with complex and variable topology. Moreover the source and destination area will not be constrained to have the same topology nor to be geometrically similar.

- Finally, matching the two curves is done through the computation of paths of minimal cost connecting the two curves. Hence the matching is not restricted to a displacement field as it is the case in most curves matching algorithms. Moreover, the method can handle changes in topology which occur when a contour is splitted into several components.

These choices will be translated within a three steps scheme based first on a specific representation of the source and destination areas, then on the computation of a similarity measure defined through a graph surface and, finally on the characterization of the geodesic paths.

\subsection{Plan}

Our approach is based on the computation of a geodesic distance map used for the definition of a similarity measure between the two curves to be matched. For this purpose, we first describe in sections 2.1 and 2.2 the computation of a geodesic on an arbitrary graph surface. In section 3, we 
explain how to define the graph surface associated to two curves of arbitrary topology in order to perform a pointwise matching of the two curves. The experimental results in section 4 illustrate the proposed method on synthetic and real images. Finally, section 5 concludes the paper and outlines some perspectives and future applications of the method to specific environmental problems.

\section{Geodesic Distance Computation}

Given two points on a surface, the computation of a geodesic joining these two points can be performed in several ways [8]. However, in this paper we consider the approach proposed by Kimmel et al [10]. Their method is based on the propagation of two closed curves surrounding each point. The geodesics are then derived from the traces of these propagated curves.

The following notations will be used in the next sections.

Given a graph surface $Z$ parameterized by $Z=(x, y, z(x, y))$ we note:

- $p=\frac{\partial z}{\partial x}$ and $q=\frac{\partial z}{\partial y}$ the partial derivatives of $z(x, y)$.

- $\vec{Z}_{x}$ and $\vec{Z}_{y}$ the partial derivatives of $Z$ with respect to $x$ and $y$. We have, $\vec{Z}_{x}=(1,0, p)$ and $\vec{Z}_{y}=(0,1, q)$.

- $\vec{N}$ the normal to the graph surface $Z$. We have,

$$
\vec{N}=\frac{\vec{Z}_{x} \times \vec{Z}_{y}}{\left\|\vec{Z}_{x} \times \vec{Z}_{y}\right\|}=\frac{(-p,-q, 1)}{\sqrt{1+p^{2}+q^{2}}}
$$

- $\alpha(s)$ is a parameterized curve on the surface $Z$ depending on the parameter $s: \alpha(s)=$ $(x(s), y(s), z(s))$.

- $\vec{T}$, the tangent to the curve $\alpha(s)$ lying on the surface is given by:

$$
\vec{T}=\frac{\overrightarrow{\alpha_{s}}}{\left|\alpha_{s}\right|}=\frac{\left(x_{s}, y_{s}, z_{s}\right)}{\sqrt{x_{s}^{2}+y_{s}^{2}+z_{s}^{2}}}
$$

where $x_{s}, y_{s}$ and $z_{s}$ are the derivatives of the curve's coordinates.

- $\alpha(s, t)$ represents the evolution of the curve $\alpha(s)$ with respect to the parameter $t$. We have $\alpha(s, 0)=\alpha(s)$. For each value of the parameter $\underline{t}$ (underlined when fixed), we obtain a new curve $\alpha(s, \underline{t})$ depending of the parameter $s$.

$\mathrm{RR} \mathrm{n}^{\circ} 3191$ 
- $\tilde{\alpha}(s, t)$ is the projection of the curve $\alpha(s, t)$ on the $(x, y)$-plane.

- $\overrightarrow{\tilde{t}}$ the tangent of $\tilde{\alpha}(s, t)$ and $\overrightarrow{\tilde{n}}$ its normal on the $(x, y)$-plane. We have: $\overrightarrow{\tilde{t}}=\frac{\left(x_{s}, y_{s}\right)}{\sqrt{x_{s}^{2}+y_{s}^{2}}}$ and $\overrightarrow{\tilde{n}}=\frac{\left(-y_{s}, x_{s}\right)}{\sqrt{x_{s}^{2}+y_{s}^{2}}}$.

\subsection{Geodesic distance}

The distance between two points on a given surface is defined through the shortest path length joining these points. This shortest path is also equivalent to the minimal geodesic [8] and is computed by the method proposed by Kimmel et al [10]. They showed that geodesic curves are the traces (i.e. points of constant curve parameter $s$ ) of the curve evolving according to the equation:

$$
\overrightarrow{\alpha_{t}}(\underline{s}, t)=\frac{\partial \alpha}{\partial t}(\underline{s}, t)=\vec{N} \times \vec{T}
$$

where, $\frac{\partial \alpha}{\partial t}(\underline{s}, t)$ represents the evolution of $\alpha(\underline{s}, t)$ at the parameter value $s, \vec{N}$ is the surface's normal and $\vec{T}$ is the tangent to the curve $\alpha(s, \underline{t})$. This equation is called the equal distance contour evolution of a curve $\alpha(s)$ on the surface $Z$.

The solution is difficult to handle for a general 3D surface, but restricting it to a graph surface simplifies the computation. Indeed, Kimmel et al [10] showed that the solution may be characterized through the projection of the curve $\alpha(\underline{s}, t)$ on the $(x, y)$-plane.

In the following of this section we derive, from equation (1) the propagation equation of the projection of the curve $\alpha(\underline{s}, t)$ on the $(x, y)$ plane (the projecting curve will be noted $\tilde{\alpha}(\underline{s}, t))$. We will also prove that this projection scheme allows to easily derive an Eulerian formulation of the projected curve's propagation in order to handle curves with complex topology and to increase accuracy and stability of the solution. 
Using the notations defined in the previous section, equation (1) can be rewritten in the following way:

$$
\begin{aligned}
\overrightarrow{\alpha_{t}}(\underline{s}, t) & =\left(x_{t}(s, t), y_{t}(s, t), z_{t}(s, t)\right) \\
& =\vec{N} \times \vec{T} \\
& =\frac{(-p,-q, 1)}{\sqrt{1+p^{2}+q^{2}}} \times \frac{\left(x_{s}, y_{s}, z_{s}\right)}{\sqrt{x_{s}^{2}+y_{s}^{2}+z_{s}^{2}}}
\end{aligned}
$$

where computation is performed at point $\alpha(s, t)$.

As we previously explained, we have to characterize the evolution on the $(x, y)$ plane of the projecting curve $\tilde{\alpha}$. In order to define the propagation equation, it is important to note that the tangential component of the curve only affects the curve's parameterization and not its shape [9]. Consequently, shape's evolution depends only on the normal component. This normal component is computed in the following.

Defining a propagation scheme for the projection $\tilde{\alpha}$ along its normal $\overrightarrow{\tilde{n}}$ amounts to derive from equation (2) a function $V$ such that

$$
\overrightarrow{\tilde{\alpha}_{t}}(s, t)=V \overrightarrow{\tilde{n}}
$$

where the function $V$ represents the propagation velocity of the curve $\tilde{\alpha}(\underline{s}, t)$ along its normal. This velocity can be obtained by computing $\vec{\alpha}_{t}(s, t) \cdot \overrightarrow{\tilde{n}}$

$$
\begin{aligned}
V & =\left(x_{t}, y_{t}, z_{t}\right) \cdot \overrightarrow{\tilde{n}} \\
& =\left[\frac{(-p,-q, 1)}{\sqrt{1+p^{2}+q^{2}}} \times \frac{\left(x_{s}, y_{s}, z_{s}\right)}{\sqrt{x_{s}^{2}+y_{s}^{2}+z_{s}^{2}}}\right] \cdot \overrightarrow{\vec{n}}
\end{aligned}
$$

By expanding the cross product and using the expression of the normal $\vec{n}$, we obtain:

$$
V=\frac{\left(y_{s}+q z_{s},-x_{s}-p z_{s}\right)}{\sqrt{1+p^{2}+q^{2}} \sqrt{x_{s}^{2}+y_{s}^{2}+z_{s}^{2}}} \cdot \frac{\left(-y_{s}, x_{s}\right)}{\sqrt{x_{s}^{2}+y_{s}^{2}}}
$$

Since the curve $\alpha$ is lying on the surface $Z$, we can compute the $z_{s}$ derivative in terms of the graph surface derivatives $p$ and $q$, using the following chain rule: $z_{s}=z_{x} x_{s}+z_{y} y_{s}=p x_{s}+q y_{s}$, and we obtain:

$$
V=\sqrt{\frac{x_{s}^{2}\left(1+p^{2}\right)+y_{s}^{2}\left(1+q^{2}\right)+2 p q x_{s} y_{s}}{\left(1+p^{2}+q^{2}\right)\left(x_{s}^{2}+y_{s}^{2}\right)}}
$$

$\mathrm{RR} \mathrm{n}^{\circ} 3191$ 
This velocity propagation can also be expressed in terms of the planar normal's components

$$
\overrightarrow{\tilde{n}}=\left(n_{1}=\frac{-y_{s}}{\sqrt{x_{s}^{2}+y_{s}^{2}}}, n_{2}=\frac{x_{s}}{\sqrt{x_{s}^{2}+y_{s}^{2}}}\right)
$$

as:

$$
V=\sqrt{n_{1}^{2} \frac{1+q^{2}}{1+p^{2}+q^{2}}+n_{2}^{2} \frac{\left(1+p^{2}\right)}{1+p^{2}+q^{2}}-n_{1} n_{2} \frac{2 p q}{1+p^{2}+q^{2}}}
$$

or

$$
V=\sqrt{a n_{1}^{2}+b n^{2}-c n_{1} n_{2}}
$$

where $a=\frac{1+q^{2}}{1+p^{2}+q^{2}}, b=\frac{1+p^{2}}{1+p^{2}+q^{2}}$, and $c=\frac{2 p q}{1+p^{2}+q^{2}}$.

Hence the propagation equation of the curve $\tilde{\alpha}(s, t)$ becomes:

$$
\overrightarrow{\tilde{\alpha}}_{t}(s, t)=\sqrt{a n_{1}^{2}+b n_{2}^{2}-c n_{1} n_{2}} \quad \overrightarrow{\tilde{n}} .
$$

This evolution scheme depends on the curve parameterization, which has to be computed at each evolution step, through the computation of the normal components $\left(n_{1}, n_{2}\right)$. However, the estimation of the normal components cannot be obtained with high accuracy when the curve becomes complex and looses smoothness, since the derivatives computation is very unstable. Furthermore, a single smooth function cannot be used for the parameterization of curves having cusps or of complex topology. Consequently, an approach independent of the parameterization must be used to represent the evolution of complex structures. For that purpose each curve $\tilde{\alpha}(s, \underline{t})$ will be represented as the zero set of a two dimensional function $\varphi$ defined on the $(x, y)$-plane. This approach is called Eulerian formulation and was introduced by Osher and Sethian [12] for crystal growth modeling. Its major advantage is the ability to handle topological changes and singularities while insuring stability and accuracy.

Given a function $\varphi$ such that its zero level set evolution tracks the curve $\tilde{\alpha}(s, \underline{t})=\varphi^{-1}(0)$, we will show in the following that its propagation is governed by the equation:

$$
\varphi_{t}=V\|\vec{\nabla} \varphi\|
$$

Indeed, the classical chain rule and equation $\left(\frac{\partial x}{\partial t}, \frac{\partial y}{\partial t}\right)=\overrightarrow{\tilde{\alpha}}_{t}$ yield:

$$
\frac{\partial \varphi}{\partial t}=\frac{\partial \varphi}{\partial x} \frac{\partial x}{\partial t}+\frac{\partial \varphi}{\partial y} \frac{\partial y}{\partial t}=\vec{\nabla} \varphi \cdot \overrightarrow{\tilde{\alpha}}_{t}
$$


Using equation (3) and the fact that the planar unit normal to any level set of a continuous function $\varphi$ can be written as $\overrightarrow{\tilde{n}}=\frac{\vec{\nabla} \varphi}{\|\vec{\nabla} \varphi\|}$, we obtain:

$$
\begin{aligned}
\frac{\partial \varphi}{\partial t}=\vec{\nabla} \varphi \cdot V \overrightarrow{\tilde{n}} & =\vec{\nabla} \varphi \cdot V \frac{\vec{\nabla} \varphi}{\|\nabla \varphi\|} \\
& =V\|\vec{\nabla} \varphi\| .
\end{aligned}
$$

Finally, by replacing $\overrightarrow{\tilde{n}}=\left(n_{1}, n_{2}\right)=\left(\frac{\varphi_{x}}{\|\vec{\nabla} \varphi\|}, \frac{\varphi_{y}}{\|\vec{\nabla} \varphi\|}\right)$ in the velocity function $V$ given by equation (4), we obtain the following evolution equation:

$$
\varphi_{t}=\sqrt{a \varphi_{x}^{2}+b \varphi_{y}^{2}-c \varphi_{x} \varphi_{y}}
$$

In this section we showed that the propagation of the projecting curve $\tilde{\alpha}$ is obtained through the level set curve $\varphi^{-1}(0)$ with $\varphi$ satisfying the evolution expressed in equations (6) and (7). This formulation allows to model variation of the curve's topology while insuring a good numerical stability and accuracy. The solution of this equation will be obtained through a monotone and conservative numerical algorithm, briefly described in the next section.

\subsection{Finite Difference Approximation}

The numerical scheme is derived from the one used for solving Hamilton-Jacobi type equations. In this approach, an explicit temporal scheme is used, while the spatial gradients are derived through the minmod method which selects the smallest slope between the forward and backward derivatives.

The minmod function is defined by:

$$
\operatorname{minmod}(a, b)= \begin{cases}\operatorname{sign}(a) \min (|a|,|b|) & \text { if } a b>0 \\ 0 & \text { otherwise }\end{cases}
$$

This minmod approach allows to bound the estimated slope by the data and consequently to keep under control the total variation of the evolving solution. The spatial gradients $\varphi_{x}$ and $\varphi_{y}$ are estimated through the following equations:

$$
\varphi_{x}\left(i h_{x}, j h_{y}\right)=\operatorname{minmod}\left(D_{x}^{+} \varphi\left(i h_{x}, j h_{y}\right), D_{x}^{-} \varphi\left(i h_{x}, j h_{y}\right)\right)
$$

$\mathrm{RR} \mathrm{n}^{\circ} 3191$ 


$$
\varphi_{y}\left(i h_{x}, j h_{y}\right)=\operatorname{minmod}\left(D_{y}^{+} \varphi\left(i h_{x}, j h_{y}\right), D_{y}^{-} \varphi\left(i h_{x}, j h_{y}\right)\right)
$$

where $h_{x}$ and $h_{y}$ are the spatial steps, and $D_{x}^{+}$and $D_{x}^{-}$(resp. $D_{y}^{+}$and $D_{y}^{-}$) are the forward and backward derivatives defined by:

$$
D_{x}^{+} \varphi=\frac{\varphi(i+1, j)-\varphi(i, j)}{h_{x}}
$$

and

$$
D_{x}^{-} \varphi=\frac{\varphi(i, j)-\varphi(i-1, j)}{h_{x}}
$$

Since the solution may develop discontinuous jumps or corners, the use of the above scheme does not provide an estimation of the norm of the normal at corners. For this reason, the estimation of the squared derivatives is obtained from an upwind conservative and monotone scheme [12, 14, 15] which is the usual scheme used in level set propagation methods [7, 11, 12]. The squared derivative is estimated by:

$$
\varphi_{x}^{2}=\max \left(D_{x}^{-} \varphi, 0\right)^{2}+\min \left(D_{x}^{+} \varphi, 0\right)^{2}
$$

or using the following scheme [10]:

$$
\varphi_{x}^{2}=\max \left(D_{x}^{+} \varphi, 0,-D_{x}^{-} \varphi\right)^{2} .
$$

The difference between these two schemes is the value obtained when the forward and backward derivatives do not have the same sign at a given point. In the first case, the value $\left|D_{x}^{-} \varphi\right|^{2}+\left|D_{x}^{+} \varphi\right|^{2}$ is considered, while in the second case the maximum between $\left|D_{x}^{-} \varphi\right|^{2}$ and $\left|D_{x}^{+} \varphi\right|^{2}$ is taken. We found the second solution more stable.

The parameters $a, b$ and $c$ representing the geometry of the graph surface $Z$ in equation (7) are computed using a central finite difference estimation of $p$ and $q$ :

$$
p_{i, j}=\frac{z_{i+1, j}-z_{i-1, j}}{2 h_{x}} \quad \text { and } \quad q_{i, j}=\frac{z_{i, j+1}-z_{i, j-1}}{2 h_{y}} .
$$

These schemes are used to perform a discrete approximation of the equal distance contour propagation described by equation (7). For the temporal variation we use a forward or explicit scheme. This gives us the following discrete approximation of equation (7):

$$
\begin{aligned}
\varphi_{i j}^{n+1} & =\varphi_{i j}^{n}+\Delta t\left(a_{i j} \max \left(D_{x}^{+} \varphi_{i j}^{n}, 0,-D_{x}^{-} \varphi_{i j}^{n}\right)^{2}\right. \\
& +b_{i j} \max \left(D_{y}^{+} \varphi_{i j}^{n}, 0,-D_{y}^{-} \varphi_{i j}^{n}\right)^{2} \\
& \left.-c_{i j} \min \bmod \left(D_{x}^{+} \varphi_{i j}^{n}, D_{x}^{-} \varphi_{i j}^{n}\right) \operatorname{minmod}\left(D_{y}^{+} \varphi_{i j}^{n}, D_{y}^{-} \varphi_{i j}^{n}\right)\right)^{1 / 2}
\end{aligned}
$$


This explicit scheme is conditionally stable, and the convergence to a stationary solution is achieved when the time step $\Delta t$ and the space steps $h_{x}$ and $h_{y}$ satisfy the Courant-Friedrichs-Lewy (CFL) condition [6]:

$$
\Delta t \leq \frac{1}{\min \left(h_{x}, h_{y}\right)}
$$

In practice, the space steps are derived from the image size and the time step is chosen accordingly to equation (10).

In order to use equation (9) for computing the geodesic distance map of a curve $\alpha(s)$ on a given surface $Z$ we have to define an initial estimate $\varphi_{0}$ such that the initial curve $\alpha$ is represented through a level set of $\varphi_{0}$. This implicit representation of the curve must satisfy the following requirement:

the function $\varphi_{0}$ has to be smooth, negative in the interior of $\varphi_{0}^{-1}(0)$ and positive otherwise. This initial estimate can be obtained in several ways according to the data. If the areas we are trying to match have to be extracted from an image, one can use a level set approach [11] and consider this solution as an implicit representation of the area of interest. On the other hand, if the curves have already been extracted we can use a signed Euclidean distance. This distance is derived from an Euclidean distance map [2] in such a way that points lying in the interior of $\varphi^{-1}(0)$ have negative values. The initial estimate is consequently defined by:

$$
\varphi_{0}(x, y)= \begin{cases}-d(x, y) & \text { if }(x, y) \in \text { interior of } \varphi_{0}^{-1}(0) \\ 0 & \text { if }(x, y) \in \varphi_{0}^{-1}(0) \\ d(x, y) & \text { if }(x, y) \in \text { exterior of } \varphi_{0}^{-1}(0)\end{cases}
$$

Given a graph surface $Z$ and an initial estimate $\varphi_{0}$ (eq. (11)) on this surface, equation (9) characterizes the distance map on the graph surface $Z$ of the area which boundary is defined by $\varphi_{0}^{-1}(0)$. In the next section we describe how we use this distance map for matching two given curves.

\section{Curves Matching}

Curves matching consists in defining a point to point correspondence between two curves. Most of the methods used in computer vision rely on geometrical constraints or deformation measure $[1,3$, 
13]. In both approaches a good representation of the curves is needed in order to derive geometrical properties or local similarity measures. These methods usually break down in the interesting cases: when a curve contains corners and cusps, or when a curve cannot be parameterized by a single smooth function or finally when the two curves to be matched have not the same topology.

In this section we present a method based on the computation of a set of paths connecting the two curves. These curves may have several connected components and different topologies. These two curves are defined as a source area $S$ and a destination area $D$. Matching the source and destination areas is done through the computation of paths connecting these regions. These paths are defined on a graph surface and minimize a cost function which measures the similarity between these two areas. This approach is particularly attractive in the absence of a reliable geometrical information and henceforth allows to match curves which undergo a large deformation or curves with a complex and variable topology. These are the main properties of the proposed approach since we do not require any parameterization of the curves nor any geometrical information.

The source and destination areas are defined as level sets of two bivariate functions $\varphi_{0}$ and $\psi_{0}$. In order to match these two areas by making use of the geodesic distance map we have to define :

- a graph surface on which the geodesic distance map of each area will be computed,

- a cost function measuring the similarity between these two areas,

- a set of paths minimizing the cost function and connecting the two areas.

These three steps are described in the following subsections.

\subsection{Definition of a graph surface}

Given two functions $\varphi_{0}$ and $\psi_{0}$ characterizing respectively the source and destination areas (see equation (11)) we compute two distance maps $\mathcal{D}_{S}$ and $\mathcal{D}_{D}$ on the graph surface $Z$ by solving the propagation equation (9). These maps are defined by the equations:

$$
\mathcal{D}_{S}=\{(x, y, \varphi(x, y))\}
$$

and,

$$
\mathcal{D}_{D}=\{(x, y, \psi(x, y))\}
$$


where $\varphi$ and $\psi$ are respectively the solutions of equation (9) with initial estimate $\varphi_{0}$ and $\psi_{0}$. The $\varphi$ and $\psi$ functions represent the distance maps on the graph surface $Z$ of the source and destination areas. The graph surface has to be the same for the computation of $\varphi$ and $\psi$ in order to define a similarity measure based on the two distance maps $\mathcal{D}_{S}$ and $\mathcal{D}_{D}$. Furthermore, in the equal distance curve evolution model given by equation (7), the initial estimate has to be on the considered graph surface $Z$. In order to satisfy these two requirements we define the surface $Z$ with the following graph:

$$
(x, y, z(x, y))=\left(x, y, \min \left(\left|\varphi_{0}\right|,\left|\psi_{0}\right|\right)\right)
$$

This surface defines the graph surface $Z$ such that, the two curves $\varphi_{0}$ and $\psi_{0}$ are zero level set and on which the distance maps $\mathcal{D}_{S}$ and $\mathcal{D}_{D}$ are computed. At each point of the $(x, y)$-plane, the $\mathcal{D}_{S}$ and $\mathcal{D}_{D}$ maps represent the geodesic distance to the source and destination area respectively.

\subsection{Definition of a similarity measure}

In our model, we define the matching of two curves through the definition of paths with minimal cost connecting these two curves. This amounts to characterize for each point $X_{S}$ on the first curve a path starting at $X_{S}$, ending at an unknown point $X_{D}$ of the second curve and having a minimal cost. Computing such a path amounts to search for an optimal path $p^{\text {opt }}$ among all the paths $p_{X_{S}}$ starting at $X_{S}$, ending on a point $X_{D}$ of the second curve and minimizing a given cost function $f(x, y)$. The path $p^{o p t}$ is then defined by:

$$
C\left(p_{X_{S}}^{o p t}\right)=\min _{p_{X_{S}}} C\left(p_{X_{S}}\right)
$$

$C\left(p_{X_{S}}\right)$ measures the cost along the path $p_{X_{S}}$, measured by:

$$
C\left(p_{X_{S}}\right)=\int_{X_{S}}^{X_{D}} f(x, y) d s
$$

where $X_{D}$ is the first point of the area $D$ attained by the path $p_{X_{S}}$.

The cost function $f$ has to characterize the similarity between the two curves. Furthermore, the matching between the source and destination area has to be symmetric in the sense that the source and the destination area may be inverted without changing the matching paths. This means that if $\tilde{p}_{X_{S}}^{X_{D}}$ denotes the optimal path connecting the points $X_{S}$ and $X_{D}$, we have the property:

$\operatorname{RR~}{ }^{\circ} 3191$ 
$\tilde{p}_{X_{S}}^{X_{D}}=\tilde{p}_{X_{D}}^{X_{S}}$. Fulfilling such a property restricts the choice of the cost function $f$, since it must define a similarity measure which depends only on the source and destination areas independently of the order in which they are considered. For this purpose we consider the cost function given by equation:

$$
f(x, y)=\varphi(x, y)+\psi(x, y)
$$

where $\varphi$ and $\psi$ are the bivariate functions defining the distance maps $\mathcal{D}_{S}$ and $\mathcal{D}_{D}$. This function

allows to compute at each point of the plane the minimal distance on the surface $Z$ defined by equation (14) to the nearest region $S$ or $D$.

A more elaborate model should also take into account the geometrical properties of the curves. For example, we can use the curvature information in a small neighborhood of the source and destination areas. Within these neighborhoods curvature information is relevant since only small deformation occurs and the curvature measure may be used to strengthen the similarity measure.

\subsection{Characterizing the matching paths}

Defining the matching paths connecting the two areas amounts to characterize for each point $X_{S}$ on the first curve the path $p^{o p t}$ (eq. (15)) connecting this point to an unknown point $X_{D}$ on the second curve. Such an approach is not straightforward, since we have to compute all paths connecting the point $X_{S}$ to the destination area $D$ and then select the one of minimal cost. It is numerically expensive and one has to store all the paths before selecting the one of minimal cost value. Instead, we use a property of the graph surfaces $\mathcal{D}_{S}$ and $\mathcal{D}_{D}$ which relates the equal distance contour to minimal paths: the minimal paths are orthogonal to equal distance contours. Given a starting point $X_{S}$, this property defines a robust method for characterizing the path of minimal cost connecting this point to a point belonging to the destination area. Such a path is characterized through its tangent vector. Since the equal distance contours are level sets of the map $\mathcal{D}_{S}+\mathcal{D}_{D}$, the tangent vector is defined by the gradient of the cost function:

$$
\nabla f=\nabla \varphi+\nabla \psi
$$

This approach gives a reliable path construction scheme: given a point $X_{S}$ on the source area, move this point in the opposite direction of the gradient of $f$ until reaching a point on the destination area. 


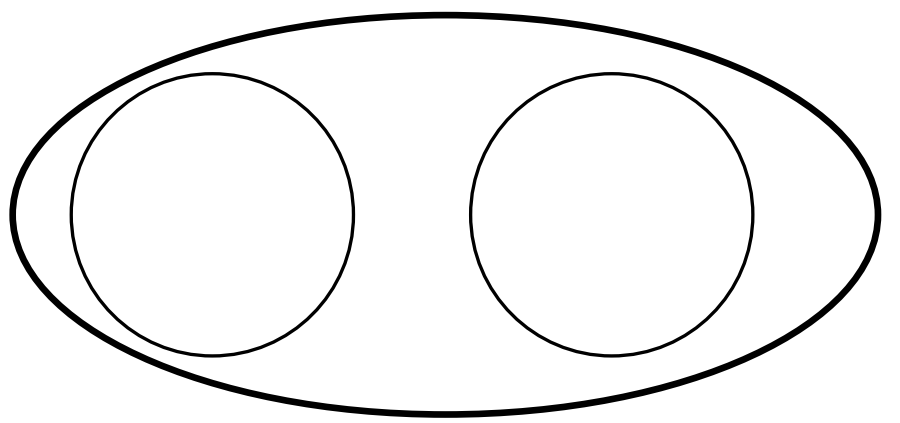

Figure 1: A plot of the curves to be matched. The source area is plotted in boldface while the two circles represent the destination area.

The result is the minimal cost path connecting the two areas and starting at $X_{S}$. This path $p(s)$ is defined by the parameterized curve $p(s)$ such that: $p(0)=X_{S}, p(1)=X_{D}$ and

$$
\frac{\partial p}{\partial s}=-\nabla\left(\mathcal{D}_{S}+\mathcal{D}_{D}\right)
$$

where $X_{S} \in \varphi_{0}^{-1}(0)$ is given and $X_{D} \in \psi_{0}^{-1}(0)$ is unknown.

\section{Experimental Results}

The proposed method is applied to synthetic data in order to test our method and real data obtained from a meteorological satellite. The first set of experimental results is given to illustrate our approach. In this case, the curves are quite simple so that the reader can figure out how the method is used. The second type of results concerns the matching of curves extracted from real Meteosat data. We will show a two frame tracking of a cloud structure in a sequence of images depicting a tropical storm.

The different experimental results given in this paper illustrate the properties of the proposed method such as complex shape deformation and matching curves based on geodesic computation. The considered data cannot be processed with the classical methods proposed by several authors [1, $3,13]$. However, matching structures undergoing a small deformation gave results comparable to the methods described in the papers [3] and [1].

$\mathrm{RR} \mathrm{n}^{\circ} 3191$ 


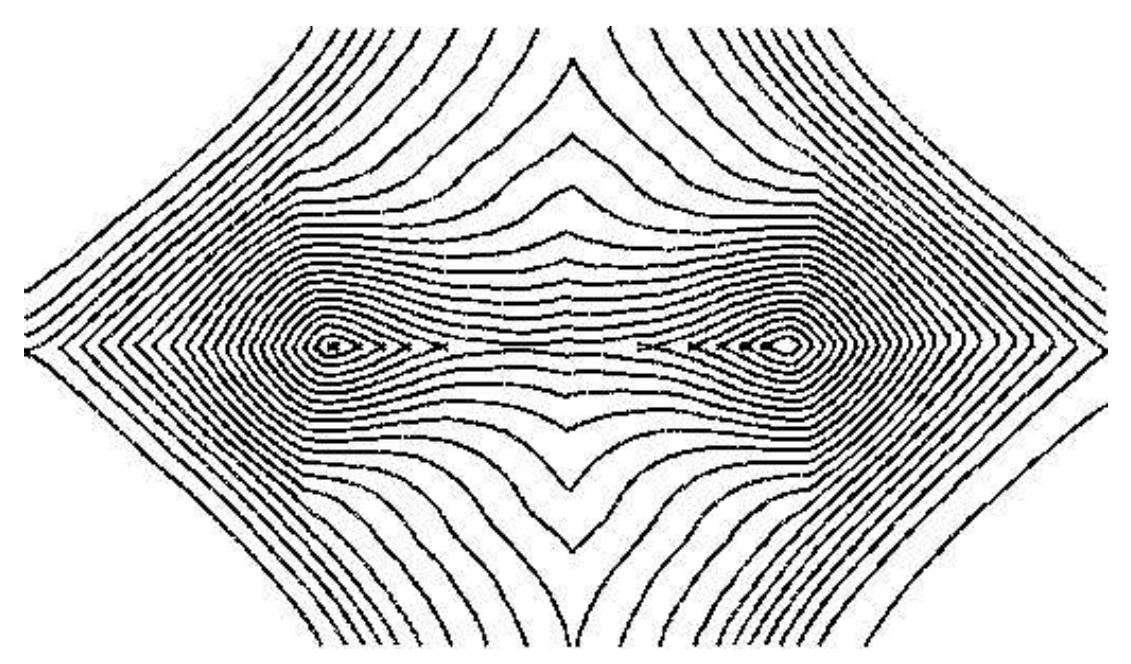

Figure 2: A level set plot of the cost function $f$ measuring the similarity between the ellipse and the circles.

\subsection{Synthetic data}

In the first synthetical example, we match an ellipse into two smaller circles. This first example is given to illustrate how the model handle curves with different topology. Figure 1 shows the curves to be matched: an ellipse into two circles. The graph surface representing the cost function which is used for path computation is displayed in figure 2.

The paths are represented in figure 3 where the blue points represent the starting points and the black ones are those located on the destination area. In this example we have a point to point matching which allows to deform one initial implicit contour into another. However, some points of the ellipse are not matched to a point on one of the circles, while every point of destination area i.e. the circles; are matched to a point located on the source area. This is due to the fact that there is none one-to-one function deforming the ellipse to the two circles.

In this example, and the following ones, we sample the source area uniformly and these points represent the source points from which the paths are computed. Although some paths are not drawn, they can be computed provided an initial point is given, since the cost function is available on the complete plan. 


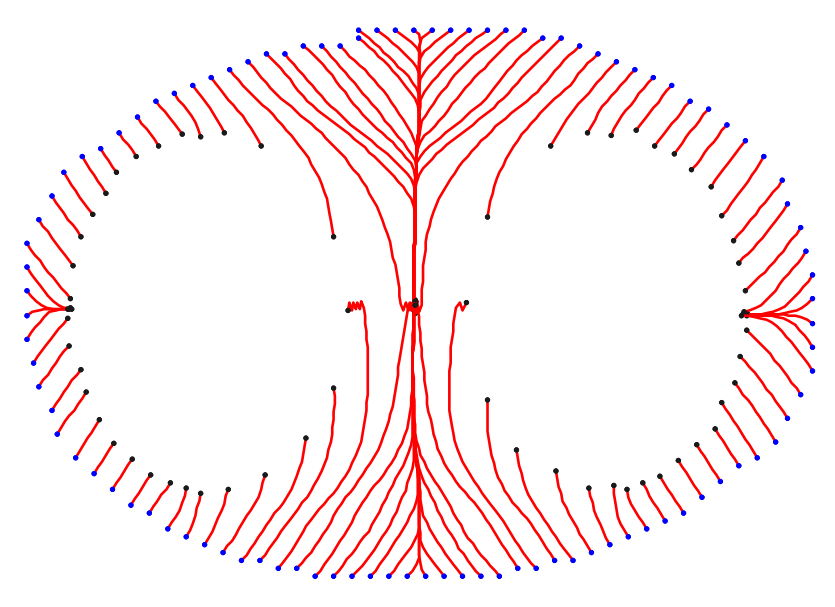

Figure 3: A plot of the paths matching the ellipse to the circles. The blue points represent the starting points of the paths and the black ones the ending points.

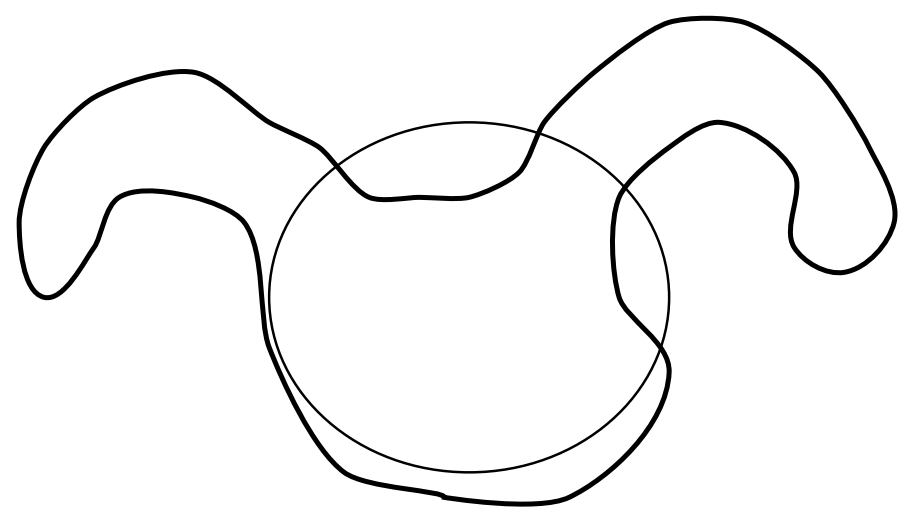

Figure 4: An example of matching curves with very large deformation. The circle go through a large deformation which yields the boldface curve.

A second example is illustrated in Figure 4. This figure shows the synthetic deformation of a circle into a complex shape. In this example, the circle undergoes a large deformation and RR n ${ }^{\circ} 3191$ 


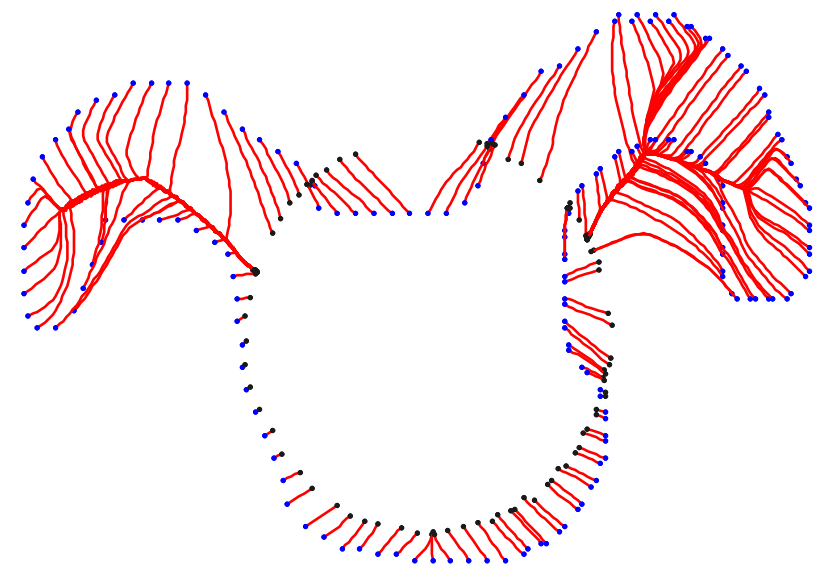

Figure 5: A plot of the paths joining the source area (blue points) to the destination area (black points). 
geometrical information like curvature is not reliable for defining a similarity measure between the two curves. Even, a method based on the surface generated by the two curves is not able to provide the paths matching the curves. Indeed, this surface has singularities since the destination area is not star shaped relatively to the source area, which means that linear paths starting from the source area do not span the complete destination area. This notion of relative star shaped curves, is one of the limitations encountered by classical matching methods which define curve matching through lines joining the two curves or minimal surfaces defined by the two contours at $z=0$ and $z=1$. In the proposed approach, we do not encounter this problem since the paths connecting the source and destination area are curves defined through the gradient of the cost function $f$ (eq. (17)). We can see in figure 5 that the paths joining the two curves do not cross one each each other. However, we observe that some paths accumulate near some regions of minimal cost since the path construction scheme is based on a gradient descent approach (see section 3.3) which characterizes such paths. Such regions do occur when a large deformation is needed to deform one curve into another one or when the topology of the curves is different.

\subsection{Meteorological images}

The last experiments were led on a Meteosat temporal sequence image of an atmospheric depression in the tropics. The Meteosat satellite have a 30 minutes acquisition's frequency. In some situations, this temporal sampling is not sufficient to characterize the structures evolution and a model have to be considered. For example, in the tropics clouds structures aggregate and disaggregate rapidly. Figure 6 shows two frames of such a sequence. In order to track this structure in a model we have to take into account that the structure deforms rapidly and its topology varies in time. The method proposed in this paper defines a new approach for curve deformation and matching using geodesic distance maps.

Figure 6 shows the result of segmentation by a snake model [5] of the two structures of interest in the Meteosat images. These two curves are considered as a source and destination area for computing the similarity function and the matching paths. These paths are plotted in figure 7 . We can observe that they are smooth and do not cross one to each other even near regions of minimal

$\mathrm{RR} \mathrm{n}^{\circ} 3191$ 

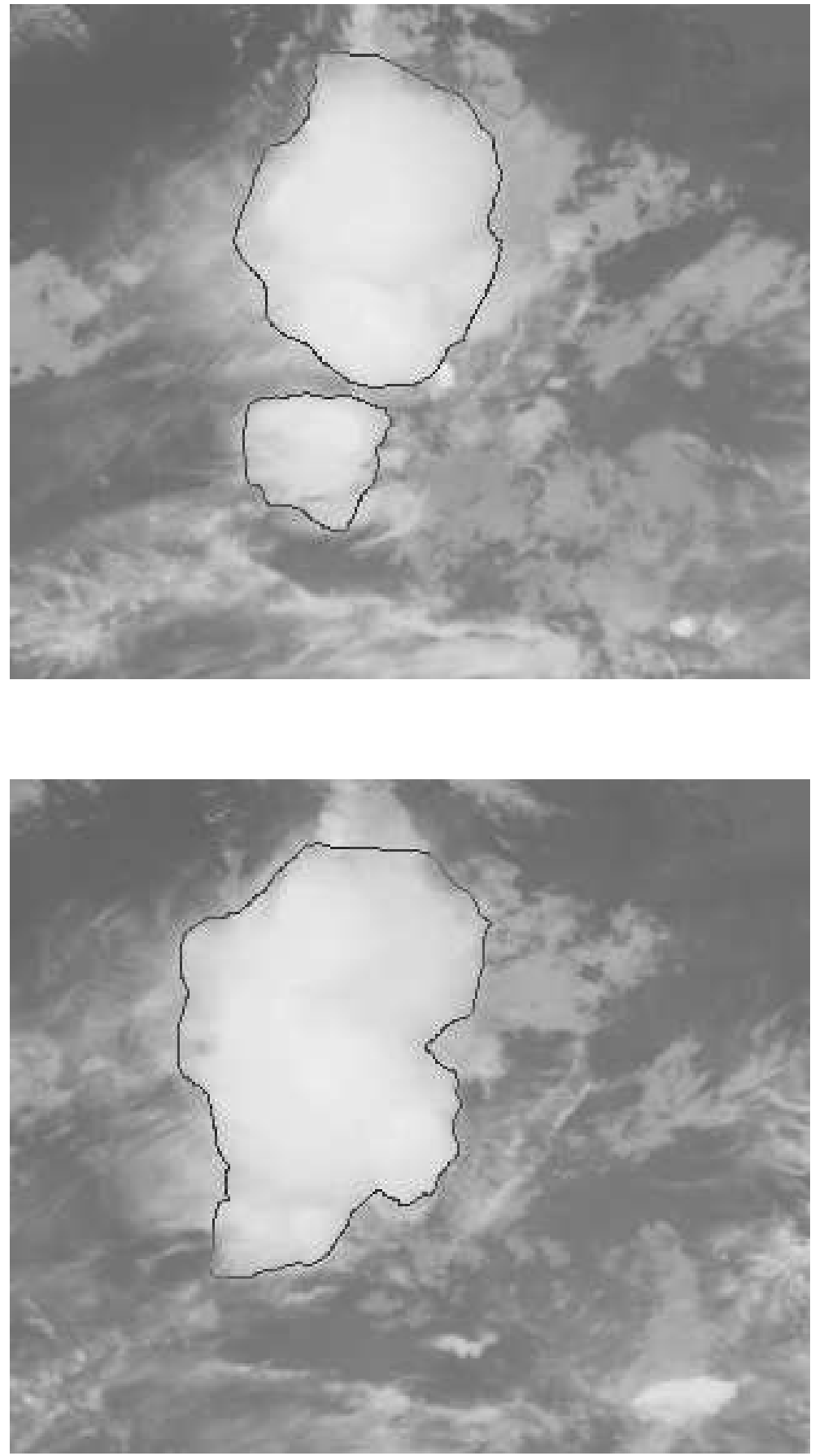

Figure 6: An extraction of clouds boundaries using a snake model in a Meteosat temporal image sequence (courtesy of LMD). These two images represent a merging of two clouds structures into one cloud. 


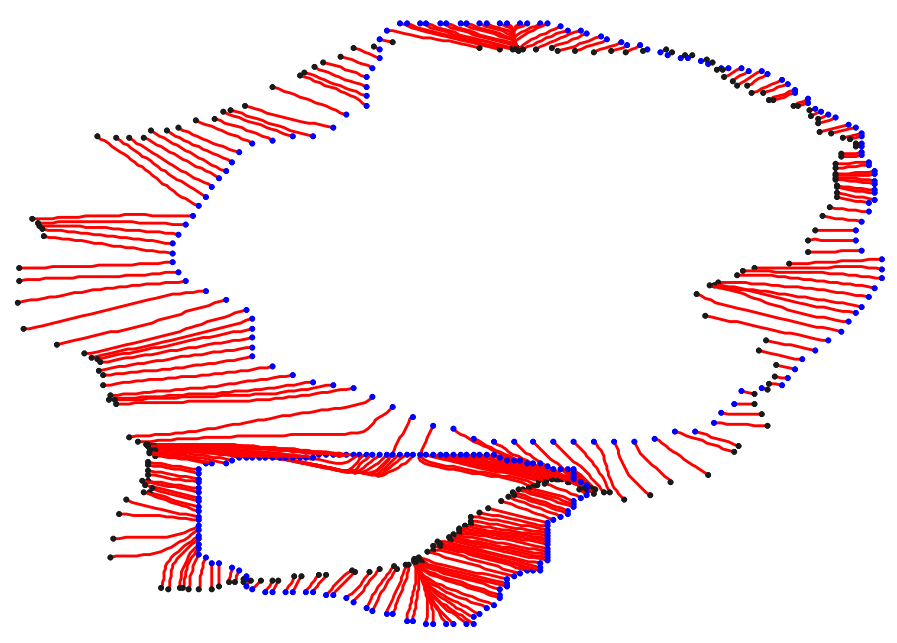

Figure 7: A plot of the paths joining the destination and source areas.

cost as we can see in figure 8 which displays a zoom of such a region. Moreover the matching paths are locally smooth and the global deformation of a structure into another one is also smooth.

\section{Conclusion and future research}

In this paper we presented a new approach for curves matching. This approach is based on the computation of paths of minimal cost connecting the two curves represented as a source and destination area. These paths lye on a graph surface measuring the similarity between the source and destination areas. This approach is particularly attractive in the absence of a reliable geometrical information and henceforth allows to match curves which undergo a large deformation or curves with a complex and variable topology. The section describing the experimental results shows that this model handle easily large deformation or topology changes. A more elaborate model is un-

RR $n^{\circ} 3191$ 


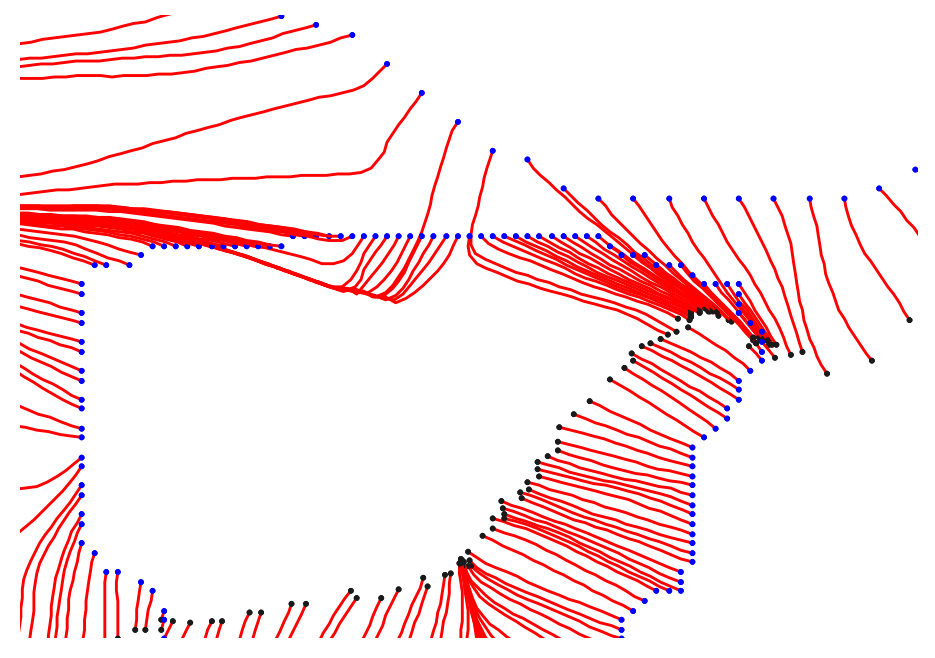

Figure 8: A zoom of a region of minimal cost where the paths connecting the two areas accumulate. We can observe that the paths do not cross one to each other and the obtained matching is consistent. 
der study in order to take into account the curvature information in a small neighborhood of the source and destination areas. Within these neighborhoods curvature information is relevant since only small deformation occurs and the curvature measure may be used to strengthen the similarity measure.

\section{References}

[1] J.P. Berroir, I. Herlin, and I. Cohen. A numerical model for large deformation on meteorological images. In International Conference on Analysis and Optimisation of Systems, Images, Wavelets and PDE's, Paris, Ministère de la Recherche, Juin 1996.

[2] G. Borgefors. Distance transformations in arbitrary dimensions. Computer Vision, Graphics, and Image Processing, 27:321-345, 1984.

[3] I. Cohen, N. Ayache, and P. Sulger. Tracking points on deformable objects using curvature information. In Proceedings of the Second European Conference on Computer Vision 1992, pages 458-466, Santa Margherita Ligure, Italy, May 1992.

[4] I. Cohen and I. Herlin. Optical flow and phase portrait methods for environmental satellite image sequences. In Proceedings of the Fourth European Conference on Computer Vision 1996, Cambridge, April 1996.

[5] Laurent D. Cohen and Isaac Cohen. Finite element methods for active contour models and balloons for 2-D and 3-D images. IEEE Transactions on Pattern Analysis and Machine Intelligence, 15(11):1131-1147, November 1993.

[6] R. Courant, K.O. Friedrichs, and H. Lewy. On the partial difference equations of mathematical physics. IBM-Journal, 11:215-235, 1967.

[7] R. Deriche, S. Bouvin, and O. Faugeras. A level-set approach for stereo. In Fisrt Annual Symposium on Enabling Technologies for Law Enforcement and Security - SPIE Conference 2942 : Investigative Image Processing., Boston, Massachusetts USA, November 1996.

$\mathrm{RR} \mathrm{n}^{\circ} 3191$ 
[8] M.P. do Carmo. Differential Geometry of Curves and Surfaces. Prentice-Hall, Englewood Cliffs, 1976.

[9] C.L. Epstein and M. Gage. The curve shortening flow. In A. Chorin and A. Majda, editors, Wave motion: Theory, modeling and Computation. Springer-Verlag, New York, 1987.

[10] R. Kimmel, A. Amir, and A.F. Bruckstein. Finding shortest paths on surfaces using levelset propagation. IEEE Transactions on Pattern Analysis and Machine Intelligence, 17(6):635640, June 1995.

[11] R. Malladi, J.A. Sethian, and B.C. Vemuri. Shape modelling with front propagation: A levelset approach. IEEE Transactions on Pattern Analysis and Machine Intelligence, 17(2):158175, February 1995.

[12] S. Osher and J.A. Sethian. Fronts propagating with curvature dependent speed: Algorithms based on Hamilton-Jacobi formulations. J. of Comput. Physics, 79:12-49, 1988.

[13] B. Serra and M. Berthod. Optimal subpixel matching of contour chains and segments. In IEEE Proceedings of the International Conference on Computer Vision, pages 402-407, Cambridge, Massachusetts, June 1995.

[14] J.A. Sethian. Level Set Methods: Evolving Interfaces in Geometry, Fluid Mechanics, Computer Vision and Materials Sciences. Cambridge University Press, 1996.

[15] J.A. Sethian and J. Strain. Crystal growth and dendritic solidification. Journal of Computational Physics, 98:231-253, 1992. 


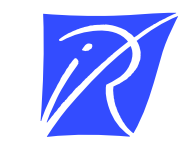

Unit'e de recherche INRIA Lorraine, Technop^ole de Nancy-Brabois, Campus scientifi que, 615 rue du Jardin Botanique, BP 101, 54600 VILLERS LÈS NANCY

Unit'e de recherche INRIA Rennes, Irisa, Campus universitaire de Beaulieu, 35042 RENNES Cedex

Unit'e de recherche INRIA Rh`one-Alpes, 655, avenue de l'Europe, 38330 MONTBONNOT ST MARTIN

Unit`e de recherche INRIA Rocquencourt, Domaine de Voluceau, Rocquencourt, BP 105, 78153 LE CHESNAY Cedex

Unit'e de recherche INRIA Sophia-Antipolis, 2004 route des Lucioles, BP 93, 06902 SOPHIA-ANTIPOLIS Cedex

\section{Éditeur}

INRIA, Domaine de Voluceau, Rocquencourt, BP 105, 78153 LE CHESNAY Cedex (France)

http://www.inria.fr

ISSN 0249-6399 